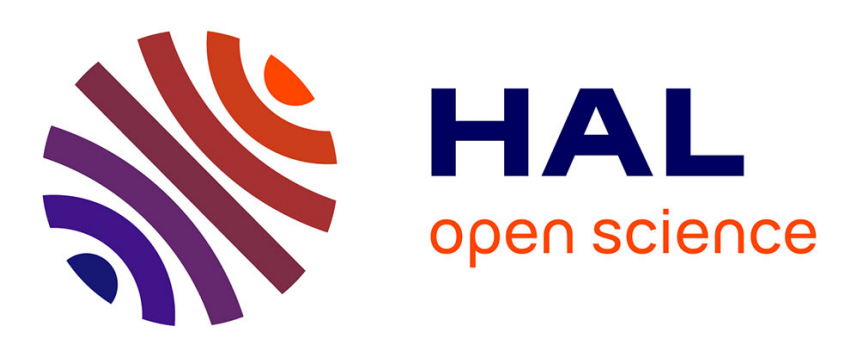

\title{
Conduites suicidaires et corticothérapie : à propos d'un cas
}

\author{
G. Carle, G. Abgrall-Barbry
}

\section{To cite this version:}

G. Carle, G. Abgrall-Barbry. Conduites suicidaires et corticothérapie : à propos d'un cas. L'Encéphale, 2016, 10.1016/j.encep.2016.01.005 . hal-01288213

\section{HAL Id: hal-01288213 \\ https://hal.sorbonne-universite.fr/hal-01288213}

Submitted on 14 Mar 2016

HAL is a multi-disciplinary open access archive for the deposit and dissemination of scientific research documents, whether they are published or not. The documents may come from teaching and research institutions in France or abroad, or from public or private research centers.
L'archive ouverte pluridisciplinaire HAL, est destinée au dépôt et à la diffusion de documents scientifiques de niveau recherche, publiés ou non, émanant des établissements d'enseignement et de recherche français ou étrangers, des laboratoires publics ou privés. 
ARTICLE IN PRESS L'ENCEPHALE

Conduites suicidaires et corticothérapie : à propos d'un cas

Corticotherapy and suicidal behavior: A case report

Auteurs : G. Carle ${ }^{a_{*}, b}$, G. Abgrall-Barbry ${ }^{a, b}$

a Département de psychiatrie, psychotraumatisme et addictions, CHU Tenon, 4, rue de la Chine, 75020 Paris, France

${ }^{\mathrm{b}}$ Université Pierre-et-Marie-Curie, 15, rue de l'École-de-médecine, 75006 Paris, France

${ }^{*}$ Auteur correspondant : HôpitalTenon, 4, rue de la Chine, 75020 Paris, France.

Adresse e-mail : guilhemcarle@gmail.com (G. Carle).

Historique de l'article : Reçu le 25 avril 2014, accepté le 21 mai 2015.

Mots clés : Tentative de suicide, Dépression, Corticoïdes, Sclérose en plaques, Intoxication médicamenteuse

Keywords: Suicide attempt, Corticosteroid, Side effect, Multiple sclerosis, Steroid-induced psychiatric disorders

http://dx.doi.org/10.1016/j.encep.2016.01.005 0013-7006/@ L’Encéphale, Paris, 2015.

Pour citer cet article : Carle G, Abgrall-Barbry G. Conduites suicidaires et corticothérapie: à propos d'un cas. Encéphale (2016),

http://dx.doi.org/10.1016/j.encep.2016.01.005 G Model ENCEP-829; No. of Pages 5 


\section{Résumé}

En 2010, environ 220000 personnes ont tenté de se donner la mort en France, soit une tentative de suicide toutes les $85 \mathrm{~s}$. Les facteurs sont nombreux, parfois iatrogènes. En moyenne, 5,7 \% des patients traités par corticothérapie sont sujets à des réactions psychiatriques sévères, mais les conduites suicidaires corticoinduites restent peu étudiées et décrites, malgré leur gravité. Nous rapportons le cas d'une patiente de 50 ans traitée par méthylprednisolone par voie parentérale pendant cinq jours pour une poussée de sclérose en plaques de forme rémittente et qui a fait une tentative de suicide par intoxication médicamenteuse volontaire $15 \mathrm{j}$ après la dernière prise de traitement. Ce geste a pu être partiellement imputé aux corticostéroïdes. Ce tableau s'inscrit dans un contexte d'épisode dépressif majeur non traité évoluant depuis deux mois. La corticothérapie semble avoir acutisé la symptomatologie et c'est au décours de son geste que l'imputabilité des corticostéroïdes a pu être discutée. Au travers de cette illustration clinique, nous discutons les critères d'imputabilité de conduites suicidaires cortico-induites, et développons l'intérêt d'un diagnostic précoce lorsqu'une corticothérapie est envisagée.

(c) L'Encéphale, Paris, 2015.

\section{Abstract}

Background. - In 2010, over 220000 people attempted suicide in France, in other words up to one suicide attempt every $85 \mathrm{~s}$. Numerous risk factors are involved, including iatrogenic factors. Data from previous retrospective studies indicate, on average, $5.7 \%$ of people treated with corticosteroids develop severe steroid-induced psychiatric disorders. Steroid-induced suicidal behavior is not explicitly specified in these studies, except for one recent descriptive study. Its prevalence remains unclear and is probably underestimated.

Case report. - We report the case of a 50-year-old woman treated by intravenous methylprednisolone for a relapsing-remitting multiple sclerosis relapse. Two weeks following a five-day intravenous treatment, she attempted suicide by prescription drug overdose at home. In the two months leading up to this event, she described symptoms of major depressive disorder which were left untreated. In retrospect, high doses of corticosteroids seem to have played a significant role in the occurrence of transient acute suicidal behavior. The patient improved significantly under antidepressant treatment and was discharged after three weeks of hospitalization.

Discussion. - This clinical case points out that corticosteroids are likely to precipitate suicidal behavior in a patient with a premorbid undiagnosed depressive disorder. Early detection of psychiatric disorders before starting corticotherapy seems to be essential. However, management strategies are not explicit enough in 
the literature to be recommended. Treatment discontinuation should not be done systematically, but tapering should be considered in combination with an antidepressant drug. A recent longitudinal study by Fardet et al. on oral corticotherapy suggests a positive correlation between steroids treatment and suicidal behavior. These results may support our clinical observation. In addition, the neurobiologic correlates may give credit to the underlying pathophysiology proposed as a potential explanation to the patient's sudden suicidal behavior after corticotherapy. Physicians and patients must be aware of possible risks of increased suicidality when undergoing corticosteroid treatment in order to prevent fatal outcomes for the patient. 


\section{Introduction}

Chaque année dans le monde, près d'un million de personnes décèdent par suicide, amenant à 16 pour 100 000 le taux global de mortalité par suicide [1]. En 2010, environ 220000 personnes ont tenté de se donner la mort en France, soit une tentative de suicide toutes les $85 \mathrm{~s}$. Si les victimes de suicide sont pour près des trois quarts des hommes, les tentatives de suicide (TS) sont plus fréquentes chez les femmes (dont $65 \%$ par le biais d'intoxication médicamenteuse volontaire (IMV)). Les $45-54$ ans sont les plus touchés par le suicide (22\%) [2] et ce pourcentage augmente avec l'âge. Près de $30 \%$ des femmes décédées par suicide l'ont fait au moyen d'une IMV [3]. Mais ces estimations récentes sont probablement minimisées, du fait d'un manque de recensement des TS.

Les idées suicidaires peuvent quant à elles parfois s'inscrire dans le cadre d'effets iatrogènes, comme avec les glucocorticoïdes. Malgré leur efficacité reconnue sur un large spectre de pathologies depuis des décennies, leur prescription requiert une surveillance clinique et paraclinique rigoureuse. Les effets secondaires sont nombreux, parmi lesquels le diabète, les infections, les troubles métaboliques (rétention hydrosodée, ostéoporose, hypertension, prise de poids. . .), ou encore les troubles cutanés, connus et recherchés en pratique. La prévalence des troubles psychiatriques varie d'une étude à l'autre, allant de 1 à $50 \%$ [4-6]. Ils se trouvent être peu décrits dans la littérature et peu dépistés en pratique clinique. La question des conduites suicidaires cortico-induites n'a fait l'objet que de quelques études et cas cliniques. À partir d'une observation clinique et des données actuelles de la littérature scientifique, nous étudions le lien entre corticothérapie et troubles psychiatriques sévères, plus particulièrement les comportements suicidaires. Nous discutons par la suite des modalités de dépistage et de prise en charge possible dans ce cas, en tenant compte des corrélats neurobiologiques en jeu.

\section{Exposé du cas de Mme $P$.}

Mme P., âgée de 50 ans, est hospitalisée en psychiatrie suite à une tentative de suicide grave par IMV. Elle n'a aucun antécédent psychiatrique personnel, notamment aucune tentative de suicide. Elle ne consomme pas de toxiques. Elle est suivie pour une sclérose en plaques (SEP), de forme rémittente, diagnostiquée il y a 6 ans suite à une faiblesse musculaire des membres supérieurs, une sensation d'éblouissement lumineux, une hypopallesthésie aux membres inférieurs (déficit proprioceptif) et une fatigabilité. Les poussées apparaissent deux fois par an environ. Elle est traitée par fingolimod à $0,5 \mathrm{mg} / \mathrm{j}$ par voie orale (immunosuppresseur sélectif indiqué en monothérapie comme traitement de fond des formes très actives de SEP rémittente-récurrente), et entre 1,5 et $2 \mathrm{mg} / \mathrm{kg} / \mathrm{j}$ de méthylprednisolone pendant 2 à $4 \mathrm{j}$ lors des 
poussées. Aucun trouble cognitif n'a été rapporté par son neurologue traitant. Elle ne relate aucun antécédent familial psychiatrique ou médico-chirurgical.

À son entrée, elle décrit une douleur morale qu'elle associe à un avenir incertain et une crainte de perdre son autonomie tout comme son indépendance vis-à-vis de ses proches. Elle dit se sentir plus émotive qu'il y a quelques mois, avec une anhédonie au premier plan. La patiente est fatiguée, anxieuse, avec des troubles du sommeil (réveil matinal précoce) et une hyporexie sans perte de poids. Son attention est partielle durant l'entretien. Elle nous dit ne pas vouloir réitérer son geste, pour ne pas faire souffrir sa filleule. Cependant, nous percevons une certaine ambivalence dans son discours, lorsqu'elle explique regretter ne pas avoir « réussi ", sans que cela n'éveille en elle la moindre émotion. Nous ne retrouvons ni idée suicidaire ni intentionnalité durant cet entretien. Elle ne présente aucun élément délirant ni dissociatif, ni de confusion mentale. Sur le plan somatique, il est noté un syndrome cérébelleux statique avec une hypopallesthésie des membres inférieurs, un discret déficit moteur des deux mains, pas de syndrome pyramidal, pas de déficit sensitif, pas d'anomalies des paires crâniennes, ni syndrome dysautonomique. Elle marche avec un déambulateur, son score Expanded Disability Status Scale (EDSS), effectué lors de sa dernière consultation en neurologie, est stable à 3,5-4 (échelle croissante de 0 à 10), et ceci depuis 2009. L'imagerie par résonance magnétique effectuée lors de sa poussée retrouve des lésions inflammatoires connues et stables au niveau cérébral (hypersignaux nodulaires en séquence T2 et FLAIR au niveau péri-ventriculaire et au niveau des deux hémisphères cérébelleux sans prise de contraste) ainsi qu'une lésion inflammatoire active au niveau du rachis (hypersignal nodulaire en séquence T2 avec prise de contraste en regard du disque intervertébral T4-T5).

La patiente est célibataire sans enfants, et a arrêté son travail de libraire depuis 2010 , date de sa mise en invalidité du fait de troubles de la marche. Isolée, elle n'a dans son entourage proche que sa filleule de 20 ans, qu'elle considère comme sa fille. Elle est la deuxième d'une fratrie de trois, n'a plus de contact avec son frère depuis de nombreuses années, mais s'est rapprochée de sa sœur depuis son diagnostic de SEP. Sa mère, âgée de 80 ans, est décrite comme ayant toujours été distante, sans notion de négligences cependant. Son père, dont elle était plutôt distante dans la relation, est décédé d'un cancer de l'œsophage fulgurant lors de la première hospitalisation de Mme P., où le diagnostic de SEP avait été posé.

Dans les mois qui précèdent son hospitalisation, MmeP. décrit un fléchissement thymique avec une peur de mourir, un pessimisme envers l'avenir, des insomnies avec difficultés d'endormissement, et des ruminations anxieuses centrées sur sa perte d'autonomie. Du bromazépam $6 \mathrm{mg} / \mathrm{jr}$ le soir est introduit par son médecin traitant. II apparaît des idées suicidaires plusieurs semaines après, avec un scénario élaboré sans passage à l'acte. Elle stocke ainsi des médicaments à son domicile pendant 1 mois. Peu après cette période, une nouvelle poussée de SEP est diagnostiquée. Elle présente alors un syndrome cérébelleux statique, une 
hypopallesthésie des membres inférieurs, un déficit moteur bilatéral des membres supérieurs à $1 / 5$, une fatigabilité et des insomnies. C'est dans ce contexte qu'elle est hospitalisée en neurologie. Mme P. reçoit alors $1,5 \mathrm{mg} / \mathrm{kg} / \mathrm{j}$ de méthylprednisolone par voie parentérale pendant cinq jours et récupère rapidement sur le plan moteur. Elle dit réagir habituellement au traitement par un état de bien-être psychique. C'est la deuxième fois qu'on lui administre une telle dose (la première cure ayant été bien tolérée). Suite à ce traitement, elle ressent un changement dans son humeur et dans son comportement à partir du troisième jour de traitement. Elle présente alors des tremblements d'action des extrémités des membres supérieurs, une tension interne avec irritabilité, une insomnie sans fatigue, une hyperphagie sans modification de sa libido, une plus grande estime d'elle-même, davantage d'énergie, une désinhibition comportementale modérée, une fuite des idées, avec cependant un fort pessimisme envers l'avenir et des idées noires. Cet état mixte évolue sur quinze jours. Le jour de son anniversaire, après un repas de famille, la patiente se retrouve seule à son domicile et décide d'ingérer cinq boîtes de bromazépam, une boîte de fingolimod, deux boîtes de métopimazine, un verre d'alcool fort, tout en plaçant proche d'elle une lame de rasoir. Aucun facteur déclenchant n'est retrouvé, en dehors du contexte de poussée de SEP traitée. Trente minutes plus tard, une voisine alerte les pompiers qui la transfèrent dans notre service d'accueil et d'urgences, puis elle est hospitalisée quelques jours plus tard en unité de psychiatrie.

La patiente explique ne jamais avoir trouvé le « courage » de se donner la mort avant cet épisode. Elle avance que cette sensation de désinhibition comportementale lui aurait permis de concrétiser son scénario. Devant ce tableau d'épisode dépressif majeur (EDM) d'intensité modérée à sévère et l'absence d'éléments hypomaniaques ou maniaques lors de son évaluation d'entrée, nous introduisons de l'agomélatine à $25 \mathrm{mg} / \mathrm{j}$. Le bilan préthérapeutique de rigueur était normal (notamment les transaminases). Nous constatons une amélioration dès j5. Elle critique son geste et n'exprime plus aucune idée suicidaire. Elle retrouve progressivement des capacités à éprouver du plaisir dans les activités et ne ressent plus aucune tension interne. Elle montre de l'entrain au quotidien, et récupère un sommeil de qualité avec l'adjonction d'alimémazine $5 \mathrm{mg} / \mathrm{j}$. Elle décrit cependant des troubles de concentration mineurs persistants, et un pessimisme envers l'avenir, du fait de sa pathologie chronique. Sur l'échelle d'humeur dépressive (EHD, auto-questionnaire évaluant l'humeur, validé chez les patients atteints de SEP), la patiente avait un score à l'entrée de 33/44, et de 19/44à J15 (à noter un score MADRS passé de 43/60 à 17/60). Un autoquestionnaire d'hypomanie de Angst a été rempli rétrospectivement, afin d'évaluer les symptômes apparus dans les jours suivant la corticothérapie (score de 7/19). À noter qu'elle n'est pas validée pour les patients atteints de SEP. Sur le plan somatique, la patiente récupère complètement au niveau moteur. Seuls persistent des troubles de la marche modérés, du fait d'une hypopallesthésie des membres inférieurs. Elle sort après 18 j d'hospitalisation pour être transférée en séjour de soins de suite et réhabilitation neurologique 
avec de l'agomélatine $25 \mathrm{mg} / \mathrm{j}$, du fingolimod $0,5 \mathrm{mg} / \mathrm{j}$, du bromazepam $3 \mathrm{mg} / \mathrm{j}$ (diminution progressive de la posologie à visée de sevrage complet), et de l'alimemazine $5 \mathrm{mg} / \mathrm{j}$. Elle accepte un suivi psychiatrique régulier.

\section{Discussion}

Dans ce cas, le diagnostic d'EDM sévère avec TS par IMV a été posé. En effet, lors de son hospitalisation dans notre service, elle ne présentait pas d'éléments hypomaniaques ou maniaques. Seuls les éléments dépressifs (suivant les critères du Diagnostic and Statistical Manual of Mental Disorders 4th Edition-TR) étaient retrouvés, avec l'absence d'antécédents psychiatriques personnels et familiaux. Nous avons donc introduit de l'agomélatine, plutôt qu'un thymorégulateur. La prescription d'un inhibiteur sélectif de la recapture de la sérotonine a été proposée en premier lieu et refusé par la patiente, malgré notre insistance. À noter que le fingolimod aurait pu également être imputé dans l'apparition initiale de l'EDM, mais dans ce cas, la prescription datant de plusieurs années était en défaveur de cette imputabilité.

Rétrospectivement, il s'est posé la question d'un trouble de l'humeur induit par corticoïdes avec caractéristiques mixtes, étant donné la présence concomitante d'éléments dépressifs et hypomaniaques à quelques jours de la corticothérapie. Cependant, l'aspect spontanément résolutif nous a incité à ne traiter que le tableau clinique dépressif actuel.

En revanche, l'amélioration clinique rapide de la patiente sur le plan thymique a nécessité une surveillance particulière quant au risque de virage maniaque. Aucun élément clinique s'y rapportant n'a cependant été retrouvé tout au long du suivi. II n'a été constaté aucune rechute thymique après un suivi d'un an.

Rétrospectivement, il a été question de déterminer l'imputabilité des corticoïdes dans l'apparition d'un potentiel suicidaire. La patiente présentait avant même son hospitalisation :

- des facteurs de risque suicidaire : un EDM évolutif, une sensation de désespoir, une pathologie chronique invalidante, un isolement social, l'absence d'activité professionnelle ;

- une dangerosité : des moyens à disposition (son traitement, un rasoir) ;

- un degré d'urgence : un scénario établi.

À cela s'est ajoutée une poussée symptomatique de SEP qui a nécessité un traitement par corticoïdes. La littérature scientifique met en avant certains facteurs permettant d'imputer ce passage à l'acte aux corticoïdes. Chez Mme P., les facteurs retrouvés sont la galénique (voie parentérale), la posologie élevée (supérieure à $80 \mathrm{mg} / \mathrm{j}$ ) et le délai d'apparition des symptômes (dans les premières semaines du traitement). L'hypothèse que nous émettons dans ce cas est celle d'un lien indirect entre le traitement et la tentative de suicide. La patiente nous décrit une sensation de bien-être et de sérénité quelques jours après l'introduction 
de la méthylprednisolone. Dans ce contexte de crise suicidaire latente, le traitement a ainsi pu jouer le rôle de catalyseur permettant de mettre en acte ses pensées.

Sur un plan plus phénoménologique, la patiente explique avoir décidé d'investir activement les soins concernant sa maladie jusqu'à sa limite personnelle " le moment où je deviendrai un légume ». Cette position défensive semble lui permettre de pallier au sentiment d'inutilité qu'elle met en avant, conséquence de ses séquelles motrices. Ne puisant que dans ses ressources psychiques personnelles dans ce combat, elle finit par s'isoler, son entourage soutenant ne se limitant qu'à sa filleule. Au travers d'un investissement fort des exercices quotidiens de rééducation physique, un sentiment de maîtrise de son corps refait finalement surface, contribuant à l'amélioration de son état physique. Cette énergie lui permet d'étouffer le sentiment de découragement et de lassitude, pour laisser place à l'espoir, l'initiative et l'action [7].

Mme P. semble organiser ses pensées par le biais de généralisations et réflexions manichéennes. Elle décrit par exemple avoir la certitude que sa vie finira inéluctablement dans une prison de chair, " l'esprit emmuré dans un corps paralysé ». Ces craintes de paralysie motrice irréversible et de perte de contrôle sont des sujets récurrents de ses ruminations. II semble en découler une appréhension forte des poussées de SEP et une forte impulsivité. Devant l'impossibilité de se projeter dans l'avenir, elle se perçoit comme dépendante à l'autre, et ne conçoit pas une telle position. Le geste suicidaire paraît s'inscrire comme l'ultime moyen de maîtrise d'une vie sans contrôle. Ainsi, un scénario suicidaire méthodiquement pensé s'est construit au fil des mois. II restait latent, jusqu'à cette dernière poussée de SEP. Les symptômes moteurs sévères apparus soudainement ont amené chez la patiente une crainte de perte d'autonomie. Marquée par cette phase temporaire de paralysie physique qu'elle redoutait tant, elle n'arrivait plus à percevoir de perspectives d'avenir. Cet épisode l'a amené à expérimenter sa propre vulnérabilité face à la mort. Dans ce contexte, l'angoisse de sa propre mort, associée à celle de la désinhibition comportementale semblent lui avoir permis de concevoir un tel geste, omettant la présence protectrice de sa filleule. L'angoisse l'emportant sur son désir de ne pas faire souffrir ses proches, Mme P. a agi dans le but, dit-elle, d'apaiser ses souffrances, et de ne pas vivre dans la dépendance totale à l'autre.

II faut également prendre en compte la pathologie chronique progressivement invalidante que présente Mme P. La SEP est une des principales causes de handicap de l'adulte jeune dans le monde, se caractérisant par un processus neurodégénératif, inflammatoire et démyélinisant du système nerveux central. La prévalence vie-entière de l'EDM dans la SEP est d'environ $50 \%$, soit 3 à 4 fois plus qu'en population générale [8]. Le potentiel invalidant des symptômes est donc au premier plan.

Finalement, le rôle des corticoïdes sur le comportement auto-agressif de la patiente semble s'inscrire dans un ensemble de facteurs ayant favorisé ce geste. Ce contexte de crise suicidaire latente et non diagnostiquée, l'EDM et cette pathologie chronique invalidante ont certainement joué un rôle important dans 
ce passage à l'acte. Le service de pharmacovigilance a été prévenu. Malgré les éléments avancés, ils ont émis des réserves concernant l'imputabilité directe des corticoïdes dans la tentative de suicide, tout en abondant dans le sens d'une possible influence de ceux-ci dans la précipitation du geste suicidaire. La déclaration a été faite selon les critères d'imputabilité de pharmacovigilance (Tableau 1).

Le comportement suicidaire induit par la corticothérapie a été mis en avant par quelques rares cas cliniques, quelques revues $[9,10]$ et quelques études observationnelles [11,12]. En moyenne, 5,7 \% des patients traités par corticothérapie seraient sujets à des réactions psychiatriques sévères [9]. Ces troubles n'apparaissent qu'en cas d'administration orale ou parentérale, et non par inhalation ou par voie cutanée [13]. La sensation d'euphorie, la dépression et les réactions psychotiques sont les troubles psychiatriques les plus fréquents [14]. Ces données sont à mettre en lien avec le risque suicidaire vie-entière chez les patients atteints de SEP qui se trouve être de deux à quatorze fois plus important qu'en population générale [15].

Rome et Braceland [16] décrivent 4 niveaux de réponse à la corticothérapie :

- le niveau 1 correspondant à un sentiment de bien-être psychique avec meilleure concentration et clarté de la pensée ;

- le niveau 2 incluant une insomnie et une activité motrice accrue. Ces deux stades concernent $60 \%$ des patients ;

- le niveau 3 touchant 25 à $30 \%$ des malades, avec anxiété sévère et variations thymiques modérées

- le niveau 4 concernant $5 \%$ des patients, avec des syndromes maniaques, dépressifs et/ou psychotiques.

Bolanos et al. retrouvent dans ces troubles psychiatriques graves un tiers de syndromes mixtes ou maniaques, un tiers de syndromes dépressifs et un tiers de syndromes psychotiques [4]. La dose délivrée est également à prendre en compte. Le programme du Boston Collaborative Drug Surveillance a enregistré une incidence de troubles psychiatriques cortico-induits variable en fonction de la posologie : 1,3\% (dose de moins de 40 mg/j), 4,6 \% (dose de 41 à $80 \mathrm{mg} / \mathrm{j}$ ), et 18,4\% (dose de plus de 80mg/j) [17]. La posologie ne semble cependant pas permettre de prédire la sévérité ni la durée des symptômes [18]. Le délai d’apparition du trouble psychiatrique est un autre des facteurs importants. II varie suivant les auteurs. Lewis et Smith décrivent un délai d'apparition des troubles d'environ 11,5 jours. Au total, $39 \%$ des cas apparaissent dans la première semaine et $62 \%$ dans les $15 \mathrm{j}$ [9]. Naber et al. retrouvent des troubles dans les trois premiers jours de traitement [19]. Hall et al. rapportent que $86 \%$ des patients présentent un trouble psychiatrique la semaine suivant l'introduction du traitement [20]. Une corticothérapie courte à forte dose aurait tendance à 
majorer l'impulsivité et la désinhibition comportementale [4]. D'autres facteurs de risques ont été identifiés, parmi lesquels l'âge, le sexe et les antécédents de prise de corticoïdes. II existerait un risque accru chez les jeunes, n'ayant jamais été exposées aux corticoïdes auparavant. Les femmes souffriraient davantage d'épisodes dépressifs, les hommes d'épisodes maniaques [11,12]. La présence d'un lupus serait également à prendre en compte [21].

En comparaison à la population générale, Fardet et al. retrouvent un risque sept fois plus important de conduites suicidaires ou de suicides sous corticothérapie. II est à noter cependant que l'incidence de ces événements est faible (19suicides et 90TS sur 372696 patients suivis) [11]. Les corticostéroïdes sont connus pour agir au niveau de l'humeur, de la mémoire et du cycle veille/sommeil. L'hyperactivité de l'axe hypothalamo-hypophyso-surrénalien (HHS) a été largement démontrée dans la littérature concernant la dépression [22,23], avec une concentration élevée de Corticotropin-Releasing Factor (CRF) intrathécale, de cortisol plasmatique, et une réponse moins efficace au test de freinage rapide à la Déxamethasone (TFD). II en découle un rétrocontrôle négatif altéré. Après traitement, l'axe HHS retrouve cependant une activité normale.

Les mécanismes endocriniens inhérents au comportement suicidaire sont en revanche plus controversés. II a été mis en évidence une hypoactivité de l'axe HHS chez des patients avec conduites suicidaires en comparaison à des patients déprimés, d'autant plus qu'ils présentent des traits anxieux et impulsifs [24-26]. On retrouve un taux élevé de CRF chez des patients décédés par suicide et avec conduites suicidaires $[27,28]$. Merali et al. rajoutent que cette augmentation est associée à une faible concentration de récepteurs CRF-R1au niveau du cortex frontal polaire [29]. Au niveau cérébral, les récepteurs aux minéralocorticoïdes (MR) permettent via le cortisol de réguler l'activité de l'axe HHS (en condition d'activité normale) et ont une forte affinité pour le cortisol endogène. Les récepteurs aux glucocorticoïdes (GR) sont eux utilisés pour réguler cette activité lors de stress aigus. Ils ont une forte affinité pour les corticostéroïdes de synthèse et une faible affinité pour le cortisol endogène. Les MR et GR sont présents au niveau du cortex préfrontal (CPF), de l'hippocampe, de l'amygdale, de l'hypothalamus ou encore de l'hypophyse. Il a été montré une diminution d'expression des GR au niveau du CPF et de l'amygdale chez des adolescents décédés par suicide [30].

D'autres suggèrent que le système sérotoninergique serait indirectement altéré. Une cortisolémie élevée induirait la synthèse de l'enzyme hépatique tryptophane pyrrolase et détournerait ainsi le tryptophane de la voie de synthèse du 5-HT. Cela réduirait le taux de sérotonine circulant dans le cerveau et augmenterait l'impulsivité, favorisant ainsi le comportement suicidaire [31,32]. Ainsi, il semblerait que les conduites suicidaires soient corrélées à un taux élevé de CRF et de cortisol, associé à un rétrocontrôle négatif de l'axe HHS altéré via la baisse de concentration des récepteurs CRF-R1 et GR. Enfin, il faut prendre en compte 
l'action globale des glucocorticoïdes sur les systèmes sérotoninergiques, dopaminergiques et cholinergiques centraux, impliqués dans la physiopathologie des troubles psychiatriques graves [33].

Le dépistage et le traitement des troubles psychiatriques cortico-induits sont des questions soulevées dans la littérature scientifique. Le test de freinage à la déxaméthasone a été étudié pour dépister les dépressions endogènes [34] mais a montré une faible sensibilité et une spécificité trop variable [35]. II pourrait être cependant un outil intéressant dans le dépistage du risque suicidaire. En effet, une étude retrouve une corrélation entre l'absence de freinage et la présence de conduites suicidaires chez des patients déprimés [36]. D'autres études ont montré un lien entre l'absence de freinage au test à la déxaméthasone et le décès par suicide $[37,38]$. D'autres marqueurs biologiques sont en cours d'évaluation.

II n'existe pas de recommandations claires concernant la prise en charge de TS cortico-induites. Débuter un sevrage progressif en corticoïdes ou atteindre la dose minimale efficace semble nécessaire [39]. Traiter l'EDM sévère est important s'il est présent, sachant que les antidépresseurs tricycliques seraient peu efficaces dans ce cas $[9,22]$. Le lithium pourrait avoir un effet protecteur face aux conduites suicidaires chez les patients unipolaires, mais n'a pas été étudié dans le cadre de trouble cortico-induit [40].

En conclusion, on ne peut qu'insister sur l'importance d'une évaluation psychiatrique avant toute corticothérapie envisagée. La France affiche un taux de suicide supérieur à la moyenne européenne avec 10,2 suicides pour 100000 habitants. Cela a évidemment un retentissement tragique sur le plan individuel, familial, sociétal et économique. Dans le cas de notre patiente, la corticothérapie à forte dose peut avoir précipité le passage à l'acte suicidaire. Les données de la littérature à ce sujet apportent des arguments supplémentaires à cette hypothèse. II serait donc intéressant de sensibiliser les soignants et patients, et de dépister les symptômes dépressifs et idées suicidaires avant l'initiation d'un tel traitement. Des autoquestionnaires simples à compléter (HAD ou EHD dans ce cas) pourraient être remis aux patients. Ils permettraient une évaluation objective, accessible pour tout médecin, orientant rapidement vers la nécessité d'un avis psychiatrique. Enfin, compte tenu de la gravité d'un tel geste, il semble important que de nouvelles études soient faites sur ce sujet afin d'évaluer au mieux son incidence et mieux définir sa prise en charge.

\section{Déclaration d'intérêts}

Les auteurs déclarent ne pas avoir de conflits d'intérêts en relation avec cet article.

\section{Références}

[1] WHO. Suicide prevention (SUPRE). WHO n.d. Consultable sur : http://www.who.int/mentalhealth/prevention/suicide/suicideprevent/en/ 
[2] Direction générale de la santé (DGS), Sous-direction «Santé des populations et prévention des maladies chroniques ». Etat des lieux du suicide en France 2013. Consultable sur: http://www.sante.gouv.fr/etat-des-lieux-du-suicide-en-france.html

[3] Bulletin épidémiologique hebdomadaire. Suicide et tentatives de suicide: état des lieux en France. N47-48 2011.

[4] Bolanos SH, Khan DA, Hanczyc M et al. Assessment of mood states in patients receiving long-term corticosteroid therapy and in controls with patient-rated and clinician-rated scales. Ann Allergy Asthma Immunol Off Publ Am Coll Allergy Asthma Immunol 2004;92:500-5.

[5] Curtis JR, Westfall AO, Allison J et al. Population-based assessment of adverse events associated with long-term glucocorticoid use. Arthritis Rheum 2006;55:420-6.

[6] Wada K, Yamada N, Sato T et al. Corticosteroid-induced psychotic and mood disorders: diagnosis defined by DSM-IV and clinical pictures. Psychosomatics 2001;42:461-6.

[7] Ruszniewski M. Face à la maladie grave. Patients, famille, soignants. Paris, Editions DUNOD, 2004.

[8] Feinstein A. Multiple sclerosis and depression. Mult Scler 2011;17(11):1276-1281.

[9] Lewis DA, Smith RE. Steroid-induced psychiatric syndromes. A report of 14 cases and a review of the literature. J Affect Disord 1983;5:319-32.

[10] Patten SB, Neutel Cl. Corticosteroid-induced adverse psychiatric effects: incidence, diagnosis and management. Drug Saf Int J Med Toxicol Drug Exp 2000;22:111-22.

[11] Fardet L, Petersen I, Nazareth I. Suicidal behavior and severe neuropsychiatric disorders following glucocorticoid therapy in primary care. Am J Psychiatry 2012;169:491-7.

[12] Barrimi M, Aalouane R, Aarab C et al. Prolonged corticosteroid-therapy and anxiety-depressive disorders, longitudinal study over 12 months. Encephale 2013;39(1):59-65.

[13] Drugs that may cause psychiatric symptoms. Med Lett Drugs Ther 2008;50:100-103; quiz p-p 2 following 104.

[14] Ling MH, Perry PJ, Tsuang MT. Side effects of corticosteroid therapy. Psychiatric aspects. Arch Gen Psychiatry $1981 ; 38: 471-7$.

[15] Pompili M, Forte A, Palermo M et al. Suicide risk in multiple sclerosis: a systematic review of current literature. J Psychosom Res 2012;73(6):411-417.

[16] Rome HP, Braceland FJ. The psychological response to ACTH, cortisone, hydrocortisone, and related steroid substances. Am J Psychiatry 1952;108:641-51.

[17] Drug-induced convulsions. Report from Boston Collaborative Drug Surveillance Program. Lancet 1972;2:677-9. 
[18] Clark LD, Bauer W, Cobb S. Preliminary observations on mental disturbances occurring in patients under therapy with cortisone and ACTH. N Engl J Med 1952;246:205-16.

[19] Naber D, Sand P, Heigl B. Psychopathological and neuropsychological effects of 8-days' corticosteroid treatment. A prospective study. Psychoneuroendocrinology 1996;21:25-31.

[20] Hall RC, Popkin MK, Stickney SK et al. Presentation of the steroid psychoses. J Nerv Ment Dis 1979;167:229-36.

[21] Ricoux A, Guitteny-Collas M, Sauvaget A et al. Troubles psychiatriques induits par la corticothérapie orale: mise au point sur la nature, l'incidence, les facteurs de risque et le traitement. Rev Médecine Interne Fondée Par Société Natl Francaise Médecine Interne 2013;34:293-302.

[22] Claes SJ. CRH, stress, and major depression: a psychobiological interplay. Vitam Horm 2004;69:117-50.

[23] Pariante CM. Depression, stress and the adrenal axis. J Neuroendocrinol 2003;15:811-2.

[24] Engström G, Alling C, Gustavsson P et al. Clinical characteristics and biological parameters in temperamental clusters of suicide attempters. J Affect Disord 1997;44:45-55.

[25] Lindqvist D, Isaksson A, Träskman-Bendz L et al. Salivary cortisol and suicidal behavior--a follow-up study. Psychoneuroendocrinology 2008;33:1061-8.

[26] Westrin A, Frii K, Träskman-Bendz L. The dexamethasone suppression test and DSM-III-R diagnoses in suicide attempters. Eur Psychiatry J Assoc Eur Psychiatr 2003;18:350-5.

[27] Arató M, Bánki CM, Bissette G et al. Elevated CSF CRF in suicide victims. Biol Psychiatry 1989;25:355-9.

[28] Westrin A, Ekman R, Regnéll G et al. A follow up study of suicide attempters: increase of CSFsomatostatin but no change in CSF-CRH. Eur Neuropsychopharmacol J Eur Coll Neuropsychopharmacol 2001;11:135-43.

[29] Merali Z, Du L, Hrdina P et al. Dysregulation in the suicide brain: mRNA expression of corticotropinreleasing hormone receptors and $\operatorname{GABA}(\mathrm{A})$ receptor subunits in frontal cortical brain region. $\mathrm{J}$ Neurosci Off J Soc Neurosci 2004;24:1478-85.

[30] Pandey GN, Rizavi HS, Ren X et al. Region-specific alterations in glucocorticoid receptor expression in the postmortem brain of teenage suicide victims. Psychoneuroendocrinology 2013. Section Discussion, para. 1.

[31] Braquehais MD, Oquendo MA, Baca-García E et al. Is impulsivity a link between childhood abuse and suicide? Compr Psychiatry 2010;51:121-9.

[32] Curzon G. Relationships between stress and brain 5-hydroxytryptamine and their possible significance in affective disorders. J Psychiatr Res 1972;9:243-52. 


\section{4}

[33] Gilad GM, Rabey JM, Gilad VH. Presynaptic effects of glucocorticoids on dopaminergic and cholinergic synaptosomes. Implications for rapid endocrine-neural interactions in stress. Life Sci $1987 ; 40: 2401-8$

[34] Carroll BJ, Curtis GC, Mendels J. Neuroendocrine regulation in depression. II. Discrimination of depressed from non depressed patients. Arch Gen Psychiatry 1976;33:1051-8.

[35] The APA Task Force on Laboratory Tests in Psychiatry. The dexamethasone suppression test : an overview of its current status in psychiatry.. Am J Psychiatry 1987;144:1253-62.

[36] Yerevanian BI, Feusner JD, Koek RJ et al. The dexamethasone suppression test as a predictor of suicidal behavior in unipolar depression. J Affect Disord 2004;83:103-8.

[37] Coryell W, Schlesser M. The dexamethasone suppression test and suicide prediction. Am J Psychiatry 2001;158:748-53.

[38] Norman WH, Brown WA, Miller IW et al. The dexamethasone suppression test and completed suicide. Acta Psychiatr Scand 1990;81:120-5.

[39] Warrington TP, Bostwick JM. Psychiatric adverse effects of corticosteroids. Mayo Clin Proc Mayo Clin 2006;81:1361-7.

[40] Coppen A. Lithium in unipolar depression and the prevention of suicide. J Clin Psychiatry 2000;61 Suppl 9:52-6.

Tableau 1 Critères d'imputabilité de pharmacovigilance appliqués au cas de Mme P.

\section{Score}

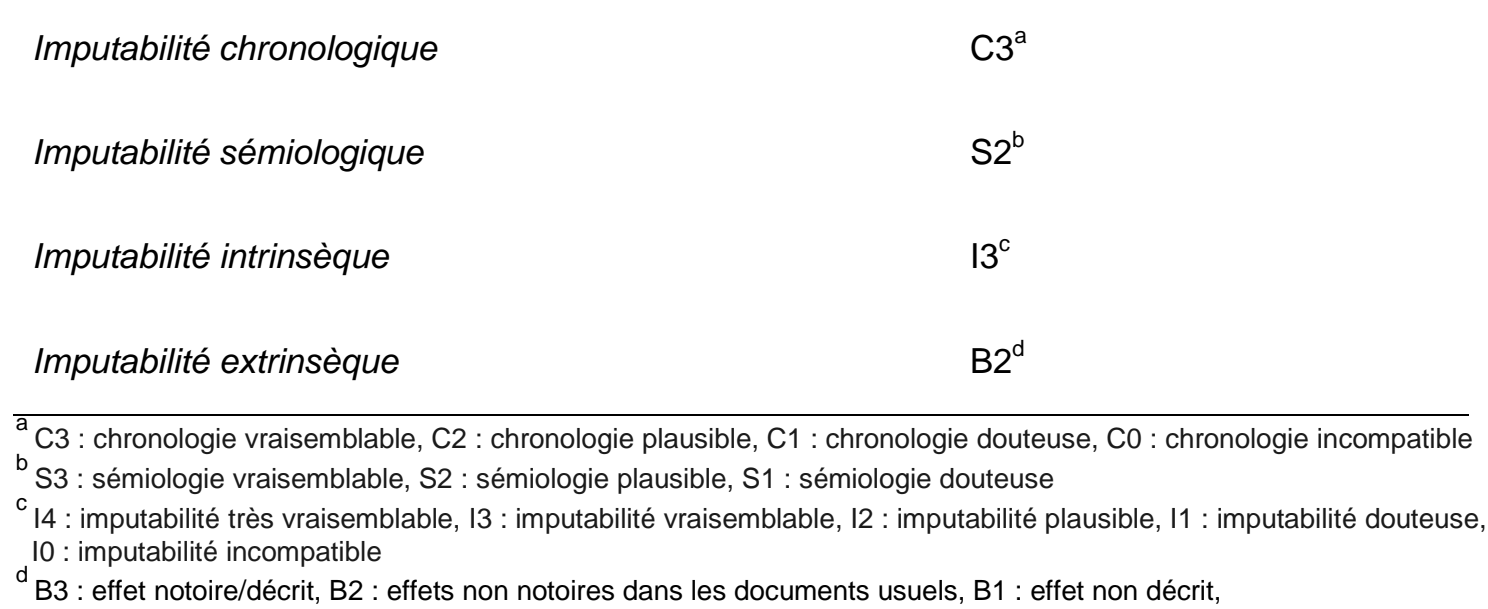

